\title{
GREENBURST: a commensal fast radio burst search back-end for the Green Bank Telescope
}

\author{
Mayuresh P. Surnis ${ }^{1,2 *}$ D. Agarwal ${ }^{1,2}$, D. R. Lorimer ${ }^{1,2}$, X. Pei $^{3}$, G. Foster ${ }^{4}$, A. Karastergiou ${ }^{4,5,6}$, \\ G. Golpayegani ${ }^{1,2}$, R. J. Maddalena ${ }^{7}$, S. White ${ }^{7}$, W. Armour ${ }^{8}$, J. Cobb ${ }^{9}$, M.A. McLaughlin ${ }^{1,2}$, \\ D.H.E. MacMahon ${ }^{9}$, A.P.V. Siemion ${ }^{9,10,11}$, D. Werthimer ${ }^{9}$ and C.J. Williams ${ }^{4}$ \\ ${ }^{1}$ West Virginia University, Department of Physics and Astronomy, P. O. Box 6315, Morgantown, WV, USA \\ ${ }^{2}$ Center for Gravitational Waves and Cosmology, West Virginia University, Chestnut Ridge Research Building, \\ Morgantown, WV, USA \\ ${ }^{3}$ Xinjiang Astronomical Observatory, Chinese Academy of Sciences, Urumqi, Xinjiang 830011, China \\ ${ }^{4}$ University of Oxford, Sub-Department of Astrophysics, Denys Wilkinson Building, Keble Road, Oxford, OX1 3RH, \\ United Kingdom \\ ${ }^{5}$ Physics Department, University of the Western Cape, Cape Town 7535, South Africa \\ ${ }^{6}$ Department of Physics and Electronics, Rhodes University, PO Box 94, Grahamstown 6140, South Africa \\ ${ }^{7}$ Green Bank Observatory, P.O. Box 2, Green Bank, WV 24944, USA \\ ${ }^{8}$ OeRC, Department of Engineering Science, University of Oxford, Keble Road, Oxford OX1 3QG, UK \\ ${ }^{9}$ Department of Astronomy, University of California, Berkeley, 501 Campbell Hall \#3411, Berkeley, CA 94720, USA \\ ${ }^{10}$ Radboud University, Nijmegen, $6525 \mathrm{HP}$, the Netherlands \\ ${ }^{11}$ SETI Institute, Mountain View, CA 94043, USA
}

\begin{abstract}
We describe the design and deployment of GREENBURST, a commensal Fast Radio Burst (FRB) search system at the Green Bank Telescope. GREENBURST uses the dedicated L-band receiver tap to search over the 960-1920 MHz frequency range for pulses with dispersion measures out to $10^{4} \mathrm{pc} \mathrm{cm}^{-3}$. Due to its unique design, GREENBURST will obtain data even when the L-band receiver is not being used for scheduled observing. This makes it a sensitive single pixel detector capable of reaching deeper in the radio sky. While single pulses from Galactic pulsars and rotating radio transients will be detectable in our observations, and will form part of the database we archive, the primary goal is to detect and study FRBs. Based on recent determinations of the all-sky rate, we predict that the system will detect approximately one FRB for every 2-3 months of continuous operation. The high sensitivity of GREENBURST means that it will also be able to probe the slope of the FRB source function, which is currently uncertain in this observing band.
\end{abstract}

Keywords: instrumentation: miscellaneous - radio continuum: transients

\section{INTRODUCTION}

Fast Radio Bursts (FRBs) are characterized by their millisecond duration and radio-frequency dispersion that far exceeds that predicted to result from interactions with free electrons in the Milky Way (Lorimer et al., 2007; Thornton et al., 2013). Over the past decade, a number of significant observations have been made, including detections from $400 \mathrm{MHz}$ (CHIME/FRB Collaboration et al., 2019a) to $8 \mathrm{GHz}$ (Gajjar et al., 2018; Zhang et al., 2018), FRBs with extreme implied distances (Bhandari et al., 2018), and the repeating sources FRB 121102 (Spitler et al., 2016) and FRB 180814 (CHIME/FRB

\footnotetext{
*E-mail: mayuresh.surnis@mail.wvu.edu
}

Collaboration et al., 2019b). The source FRB 121102 has been associated with a dwarf galaxy at redshift $z=0.19$ (Marcote et al., 2017) and shows extreme Faraday rotation (Michilli et al., 2018), demonstrating that it is situated in a dense region in its host galaxy.

FRBCAT $^{1}$ (Petroff et al., 2016) provides an up-to-date catalog of reported FRB detections with 65 reported FRBs at the time of writing this paper. Recently, there has been a significant increase in the number of reported FRBs as new wide field of view (FoV) arrays have began operating. The Australian Square Kilometre Array Pathfinder (ASKAP), when observing in 'fly's eye' mode

\footnotetext{
${ }^{1}$ http://frbcat.org
} 
to maximize sky coverage, has detected 20 FRBs (Shannon et al., 2018). Initial observations with Canadian Hydrogen Intensity Mapping Experiment (CHIME) have also resulted in 13 FRB detections (CHIME/FRB Collaboration et al., 2019a) including the repeating source FRB 180814 (CHIME/FRB Collaboration et al., 2019b).

Prior to ASKAP and CHIME, most of the FRBs were detected with the 64-m Parkes radio telescope. Only two FRBs have been detected with the Robert C. Byrd Green Bank Telescope (GBT). The first, FRB 110523 (Masui et al., 2015), was detected in archival GBT data acquired with the the prime focus $800-\mathrm{MHz}$ receiver (700-900 MHz). This detection - with a telescope other than Parkes - provided strong evidence that FRBs were in fact astrophysical. Recently, multiple bursts from the repeating source FRB 121102 were detected (Gajjar et al., 2018; Zhang et al., 2018) using the GBT C-Band $(4-8 \mathrm{GHz})$ receiver, while the Green Bank Northern Celestial Cap (GBNCC) Pulsar Survey at $350 \mathrm{MHz}$ reported a non-detection of FRBs (Chawla et al., 2017). Recently, Golpayegani \& Lorimer (2019) also reported non-detection of FRBs at L-band using the $20 \mathrm{~m}$ telescope on the GBT site.

Wide FoV arrays have been very successful in detecting FRBs, but there still exists a strong motivation for using high-gain, single-element telescopes for FRB surveys. Though the GBT has a narrow FoV compared to that of ASKAP or Parkes, the GBT provides a significant increase in sensitivity, allowing for the detection of low-fluence FRBs that would otherwise be missed. These detections would provide an important contribution to future population studies.

Based on previous detections, FRBs show no preferred sky direction. As such, commensal data acquisition systems provide augmented science output from an observation at the additional cost of running dedicated hardware (Foster et al., 2018). For a fixed FoV, maximizing the total observing time also maximizes the event detection rate. A dedicated splitter for the L-band receiver was recently installed on the GBT. This allows for fulltime observations using this receiver even when other receivers are at the Gregorian focus. These observations at lower, but stil reasonable, sensitivity still provide vital time on the sky, hence maximizing the possibility of an FRB detection.

In this work we discuss the design and implementation of the commensal FRB search back-end using the dedicated L-band tap. We discuss the signal path and processing pipeline in $\S 2$. Initial test observations to verify the pipeline are covered in $\S 3$. We also discuss the survey sensitivity and expected detection rates in $\S 4$.

\section{SYSTEM DESCRIPTION}

GREENBURST is inspired from its predecessor at the Arecibo Telescope, SETIBURST (Chennamangalam et al., 2017), and works in parallel with a duplicate SERENDIP VI system at the GBT. Although the concept for GREENBURST is derived from SETIBURST, it differs in implementation significantly. The back-end consists of three sub-systems, the commensal modifications at the front-end, the ROACH2 Field Programmable Gate Array (FPGA) board ${ }^{2}$ for signal processing, and an FRB search system. We describe each of these in the subsections below.

\subsection{L-band Receiver Commensal Modifications}

The GBT has an unblocked aperture with an off-axis arm containing an eight-position feed turret. The frontend analogue electronics are situated below a secondary reflector, $8 \mathrm{~m}$ in diameter. A circular turret with eight positions is used to select the primary observing receiver. In an earlier test, Maddalena (2013) found that apart from the focus position, the L-band (1-2 GHz) feed can be used for commensal observing in four more positions with decreasing sensitivity relative to the focus position. We have added a directional coupler to the L-band feed to allow commensal mode observations even when the feed is not being used as the primary observing feed.

The dual-polarization L-band Radio Frequency (RF) signal received from the feed (denoted as X and $\mathrm{Y}$ in Fig. 1) travels through the front-end electronics which consists of an optional circular polarization synthesizer, Low-Noise Amplifier (LNA), noise calibrator, and a userselectable notch filter. ${ }^{3}$ We installed two directional couplers (one for each of the polarizations) with $20 \mathrm{~dB}$ gain in the L-band signal chain between the notch filter and the intermediate frequency (IF) system (see Fig. 1). The output from the coupler is then transmitted to the equipment room through an optical fibre and digitized. We added isolators before the couplers to minimize reflections which were inducing ripples across the receiver bandpass.

We then conducted tests to ensure that the additional components do not affect regular observations. In these tests, we observed in the direction of the North celestial pole at night, under good weather. This reduced the possibility of introducing changes in spillover from ground, elevation/atmosphere, the sky, and baseline shapes from standing waves in the optics due to solar illumination. We conducted an 11-minute observation using the digital continuum receiver (DCR) to cover the entire L-band frequency range of $1150-1730 \mathrm{MHz}$. The addition of the coupler resulted in a negligible change in system temperature. We also conducted 40-minute observations in the single window spectral mode at centre frequencies of $1365,1400,1420$ and $1665 \mathrm{MHz}$ with a bandwidth

\footnotetext{
${ }^{2}$ https://casper.berkeley.edu/wiki/ROACH2

${ }^{3}$ The notch filter is a user-selectable filter to suppress a known radar signal in the frequency band between $1200-1310 \mathrm{MHz}$
} 


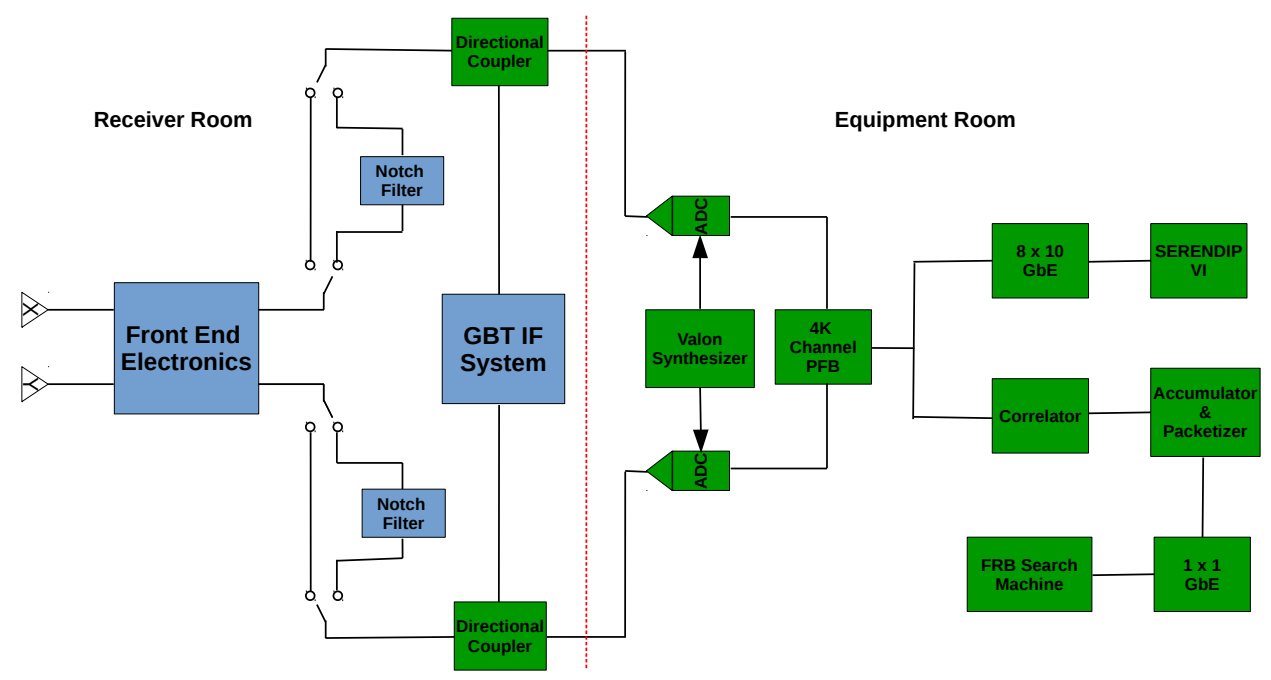

Figure 1. Block diagram showing the signal chain for the regular (blue components) and commensal mode (green components) for the L-band receiver. The signal chain on the left of the dashed red line is located in the receiver room, while the chain on the right is located in the equipment room. The signal travels on an optical fiber from the receiver room to the equipment room.

of $20 \mathrm{MHz}$ to ensure that the spectral-line data quality did not suffer due to the insertion of the couplers. We concluded that the insertion of the couplers in the signal chain did not degrade the data quality.

\subsection{Modified SERENDIP VI FPGA Design}

A ROACH2 board is used to perform digital signal processing (DSP) on the L-band signal. This board is primarily used for the SERENDIP VI system (see Chennamangalam et al., 2017, for more details) but we have updated the firmware with additional capabilities for GREENBURST. The analogue signal digitized with an 8-bit Analog-to-Digital Converter (ADC) at 1920 Msps. A Valon frequency synthesizer is used as the sampling clock. The second Nyquist zone is used to sample the 960$1920 \mathrm{MHz}$ band. A polyphase filterbank (PFB) is used to channelize the band into 4096 frequency sub-bands. The sub-bands are then power detected and accumulated to produce a spectrum every $256 \mu$ s (see Fig. 2 for an example). The spectrum is then packetized and transmitted as User Datagram Protocol (UDP) packets over a 1 gigabit Ethernet link to the FRB search machine. A complete spectrum is divided into 16 packets. Each packet contains 256 spectral channels for each polarization with a total of 768 bytes of data (256 bytes header, 256 bytes power of $\mathrm{X}$ and 256 bytes power of $\mathrm{Y}$ ). This results in a data rate of $384 \mathrm{Mbps}$. The FPGA design is publicly available ${ }^{4}$.

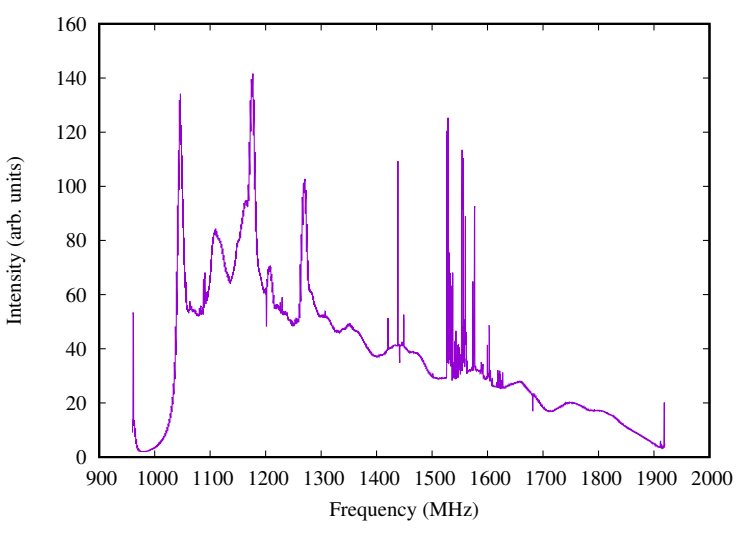

Figure 2. Bandpass response of the GREENBURST system from an observation carried out when the L-band feed was at the Gregorian focus. The bandpass shape is similar at other turret positions but with noise statistics proportional to $T_{\text {sys }}$.

\subsection{FRB Search Machine}

The FRB search machine is composed of two Intel Xeon E5-2640 processors, an NVIDIA GeForce GTX Titan Xp graphics processing unit (GPU) with Pascal architecture and 12 GB memory, a 1.2 TB solid-state drive for temporary storage and a $10 \mathrm{~TB}$ additional hard drive for long-term storage.

We use a custom receiver code ${ }^{5}$ to capture the incoming UDP packets and reconstruct the spectra. To do this, we use two static buffers, each of length $2^{17}$ samples. This corresponds to 33.6 seconds of time, which is the dispersion delay across our band at a DM of 10000 $\mathrm{pc} \mathrm{cm}^{-3}$. The static buffers are used in an alternate

\footnotetext{
${ }^{5}$ https://github.com/mpsurnis/greenburst 
fashion, i.e. at a given time, one buffer is used to save a copy of the incoming spectra while the other is used to write the previous data block to disk. The code writes $2^{21}$ spectra (corresponding to an integration time of $\sim 537 \mathrm{~s}$ ) to a single filterbank file. In order to avoid losing candidate information in the last data block, we have implemented an overlap of 33.6 seconds between two consecutive filterbank files. Once a filterbank file is created, the search pipeline carries out a single-pulse search covering a Dispersion Measure (DM) range of $10-10000 \mathrm{pc} \mathrm{cm}^{-3}$. This search is performed using the GPU-accelerated HEIMDALL program. The pipeline then sifts through potential candidates and produces plots for most likely FRB candidates. Further details of the completed pipeline will be discussed in a future paper.

\section{INITIAL OBSERVATIONS AND SENSITIVITY TESTS}

We measured the sensitivity of GREENBURST by switching between the eight turret feed positions and observing the same calibrator source. We measured the effective system temperature $T_{\text {sys }}$ for the L-band receiver using the DCR at $1375 \mathrm{MHz}$ with a $20 \mathrm{MHz}$ bandwidth and a sampling time $200 \mathrm{~ms}$ to observe the calibrator source $3 \mathrm{C} 147$. The additional noise $\Delta T$ sys due to the turret position offset (relative to the L-band feed at the focus) is plotted as black dots in Fig. 3 and listed in Table 1 . The beam shape of the feed is slightly broadened at the offset turret positions due to axial de-focusing. We measured this for each turret position using the calibrator scans. We list the turret frame offsets and measured FoV (equivalent width at the half power point) of the L-band feed in Table 1. Observing with the L-band feed when in neighbouring turret positions (C-band, MUSTANG) results in approximately $30 \%$ decrease in sensitivity. In the worst case, when the S-band feed is at the focus, the effective sensitivity of the L-band system is equivalent to a single dish with a diameter of about $28 \mathrm{~m}$.

Fig. 3 also shows the typical percentage usage of the feeds based on 2018 usage statistics (T. Minter, private communication). Turret positions 4 (ARGUS), 5 (Sband), and 6 (K-band) are partially blocked by the primary focus structure resulting is significantly reduced sensitivity. But, only approximately $15 \%$ of the 2018 observing time was used for observations with these feeds. Nearly half of the observing time was using the L-band feed, as such most GREENBURST observations will be at the optimal sensitivity.

To test the FRB search pipeline, we observed PSR B0329+54. An example candidate plot from the real-time single pulse search pipeline is shown in Figure 4. Our single-pulse search pipeline will produce similar plots for all the potential FRB candidates.

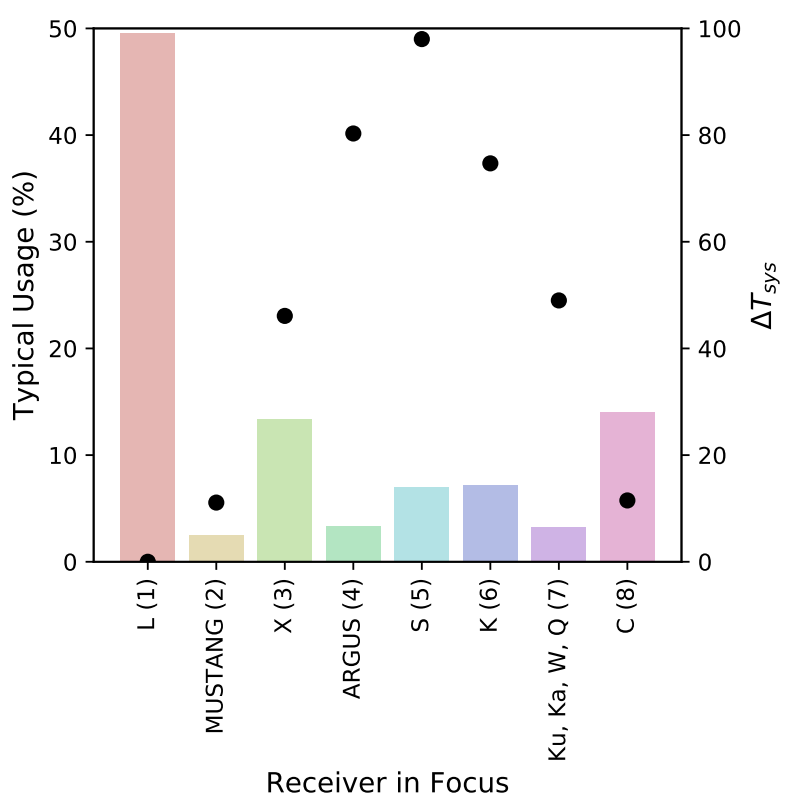

Figure 3. Excess system temperature $\Delta T_{\text {sys }}$ as a function of the receiver in focus/turret position for the GBT L-band receiver (black points). Expected typical receiver usage based on 2018 usage (bars).

\section{EVENT RATE PREDICTIONS}

To estimate the expected FRB rate with GREENBURST, we first use the radiometer noise considerations to compute the minimum detectable flux density

$$
S_{\min }=\frac{S / N_{\min } T_{\mathrm{sys}}}{G \sqrt{n_{p} t_{\mathrm{obs}} \triangle f}},
$$

where we assume a system temperature $\left(T_{\text {sys }}\right)$ of $40 \mathrm{~K}$ (including a cold sky temperature of $10 \mathrm{~K}$ ), a cut-off signal to noise ratio $(\mathrm{S} / \mathrm{N})$ of $10\left(S / N_{\text {min }}\right)$, telescope gain $(G)$ of $2 \mathrm{~K} \mathrm{Jy}^{-1}$, with 2 polarizations summed $\left(n_{p}=2\right)$ over a bandwidth $(\triangle f)$ of $960 \mathrm{MHz}$ and a typical FRB pulse width $\left(t_{\mathrm{obs}}\right)$ of $5 \mathrm{~ms}$. This results in an $S_{\min }$ of 65 mJy at the bore-sight or $130 \mathrm{mJy}$ at the half power point of the beam. Re-arranging Equation 6 from Lawrence et al. (2017), replacing $a$ by $R_{0}$ and $b$ by $\alpha$, above a flux density $S$, we get the expected FRB rate

$$
R(>S)=\frac{\pi r^{2}}{\alpha \ln (2)} R_{0}\left(\frac{S}{1 \mathrm{Jy}}\right)^{-\alpha},
$$

where $R_{0}$ is the all sky rate of FRBs and $\alpha$ is the (currently uncertain) slope of the source count distribution. For a population of sources uniformly distributed in Euclidean space, $\alpha=1.5$. We have also assumed a circular beam with a Gaussian power pattern with $r$ being the half width at half maximum. Adopting a value of $4.6^{\prime}$ 
Table 1 A summary of the relevant parameters for all GBT turret positions. From left to right, the columns list turret position, feeds currently in position, approximate turret rotation angle corresponding to the position offset from the L-band position, the offsets in azimuth and elevation, the excess system temperature for the L-band receiver as compared to the focus position, the measured FoV of the telescope beam, the antenna temperature, aperture efficiency, telescope gain, estimated sensitivity at the half-power point, usage based on total on-sky time in 2018 and our estimated FRB rate (see text).

\begin{tabular}{ccccccccccccc}
\hline \hline $\begin{array}{c}\text { Turret } \\
\text { position }\end{array}$ & Receiver & $\begin{array}{c}\text { Rotation } \\
\text { angle }\left({ }^{\circ}\right)\end{array}$ & $\begin{array}{c}\Delta \mathrm{Az} \\
\left({ }^{\prime}\right)\end{array}$ & $\begin{array}{c}\Delta \mathrm{El} \\
(')\end{array}$ & $\begin{array}{c}\Delta T_{\text {sys }} \\
(\mathrm{K})\end{array}$ & $\begin{array}{c}\text { FoV } \\
(')\end{array}$ & $\begin{array}{c}T_{\mathrm{A}} \\
(\mathrm{K})\end{array}$ & $\eta$ & $\begin{array}{c}\mathrm{G} \\
(\mathrm{K} / \mathrm{Jy})\end{array}$ & $\begin{array}{c}S_{\min } \\
(\mathrm{Jy})\end{array}$ & $\begin{array}{c}\text { Usage } \\
(\%)\end{array}$ & $\begin{array}{c}\text { FRB rate } \\
\left(\mathrm{yr}^{-1}\right)\end{array}$ \\
\hline 1 & L-band & 0 & 0.0 & 0.0 & 0.0 & 9.2 & 37.45 & 0.70 & 2.0 & 0.130 & 41.7 & $2-6$ \\
2 & MUSTANG & 300 & 22.5 & -13.2 & 11.1 & 9.4 & 28.06 & 0.52 & 1.4 & 0.236 & 2.1 & $0.05-0.2$ \\
3 & X-band & 260 & 25.3 & -30.2 & 46.1 & 9.5 & 14.01 & 0.26 & 0.7 & 0.794 & 11.2 & $0.1-0.3$ \\
4 & ARGUS & 220 & 16.2 & -45.7 & 80.3 & 10.8 & 8.26 & 0.15 & 0.4 & 1.936 & 2.8 & $0.01-0.04$ \\
5 & S-band & 180 & 0.0 & -51.5 & 98.0 & 10.8 & 4.28 & 0.08 & 0.2 & 4.454 & 5.9 & $0.01-0.04$ \\
6 & K-band & 140 & -16.9 & -45.2 & 74.7 & 10.3 & 6.97 & 0.13 & 0.4 & 1.856 & 6.0 & $0.02-0.08$ \\
7 & Ku-band $^{\dagger}$ & 100 & -25.4 & -29.8 & 49.0 & 9.6 & 14.94 & 0.28 & 0.8 & 0.718 & 2.7 & $0.02-0.08$ \\
8 & C-band & 60 & -22.1 & -12.5 & 11.5 & 9.3 & 28.84 & 0.54 & 1.5 & 0.224 & 11.8 & $0.3-1.0$ \\
\hline \hline
\end{tabular}

$\dagger$ Turret position 7 also houses Ka-band, W-band and Q-band feeds in rotation.
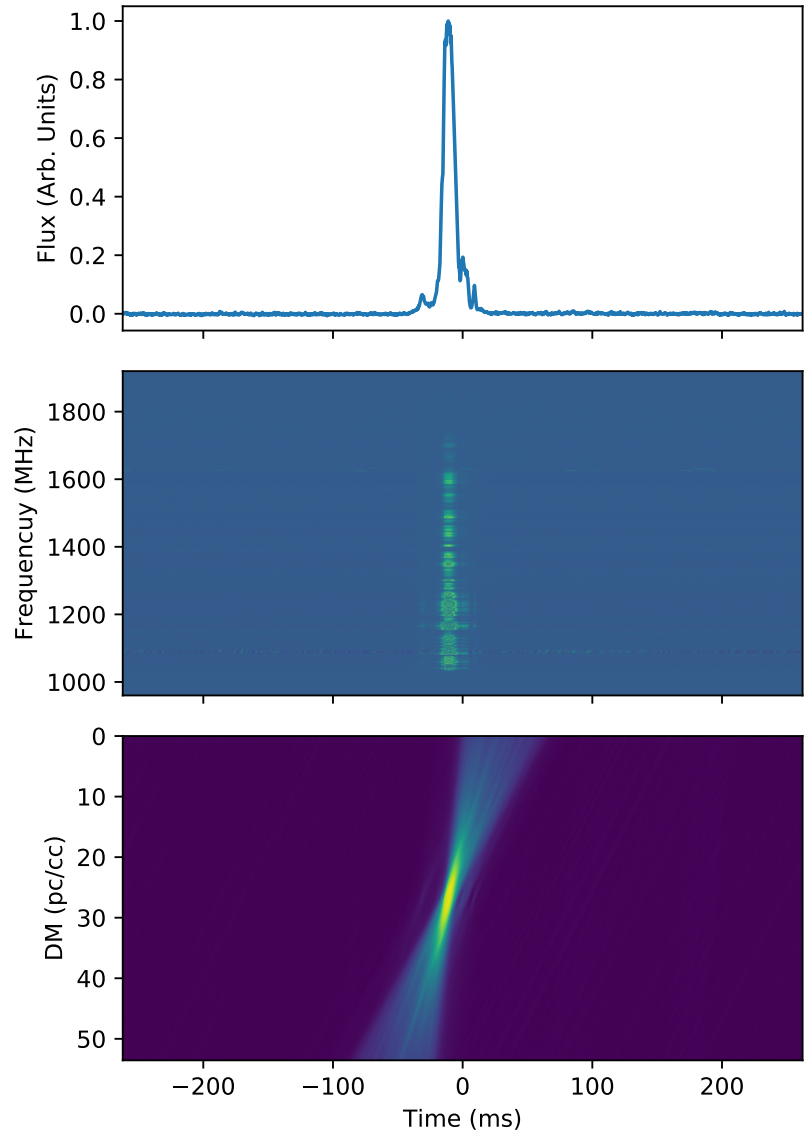

Figure 4. Candidate plot showing a single pulse detected from PSR B0329+54 with the GREENBURST pipeline. Top panel shows the pulse in time, middle panel shows the de-dispersed pulse as a function of frequency, and the bottom panel shows the pulse in DM-time plane.

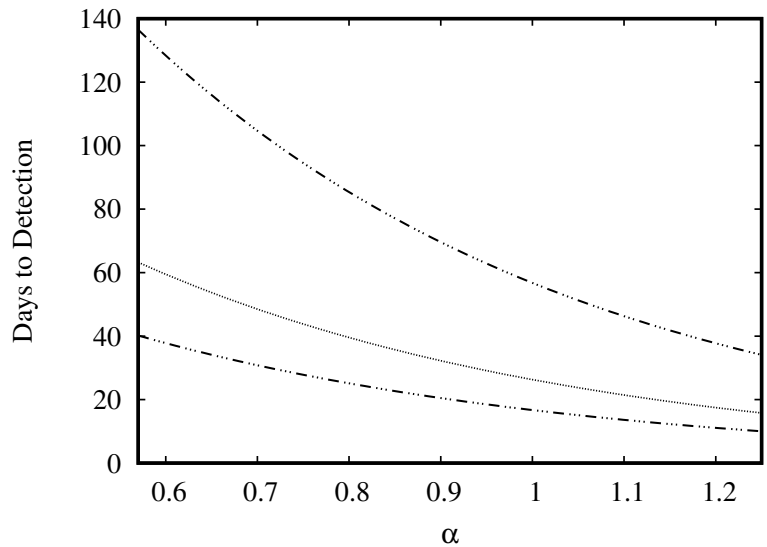

Figure 5. Predicted number of days to the first FRB detection with GREENBURST as a function of $\alpha$. The dotted line in the middle denotes the mean while the dot-dashed lines denote the $95 \%$ confidence intervals

for $r$ (see Table 1), 0.91 for $\alpha$ and a maximum sky rate $R_{0}$ of $924 \mathrm{FRB}$ events per sky per day (Lawrence et al., 2017) results in a maximum rate of $5 \times 10^{-2} \mathrm{FRB}$ events per day for GREENBURST. Inverting the rate gives us the minimum wait time of 20 days before the first FRB detection. Figure 5 shows the ranges of the wait times based on the $95 \%$ confidence intervals on $\alpha$ and $R_{0}$ as reported by Lawrence et al. (2017). For a mean value of 0.91 for $\alpha$ and 587 FRB events per sky per day for $R_{0}$ (as reported by Lawrence et al., 2017), we get a mean wait time of 32 days of continuous operation at the Gregorian focus (turret position 1) for GREENBURST. Using the method described above, we have estimated $S_{\min }$ and the projected FRB rates for other turret positions in Table 1. We expect a combined expected FRB rate of $2-7$ per year of operation. 


\section{DISCUSSION}

We have estimated the wait time for the first FRB detection adopting the all-sky rate from Lawrence et al. (2017). Based on the usage statistics for the GBT in year 2018, the L-band feed was used for scientific observations for approximately 2970 hours out of a total of about 7125 hours of on-sky time (T. Minter, private communication). For the $95 \%$ confidence interval range of the all-sky FRB rates (Lawrence et al., 2017), we project that GREENBURST would detect between 2-6 FRBs per year. This rate estimate includes the time L-band was used at the Gregorian focus. The adjacent turret positions (position 2 and 8) constitute an additional 1000 hours of observing time. With an $11 \mathrm{~K}$ excess $\Delta T_{\text {sys }}$, this would result in one additional FRB per year. The remaining time at other turret positions may result in more serendipitous detections, especially of low-DM and high-brightness events such as the recently discovered ASKAP (Shannon et al., 2018) and CHIME (CHIME/FRB Collaboration et al., 2019a) FRBs. Given this rate, even a non-detection would be useful in constraining the value of $\alpha$ towards the higher end in Figure 5. Assuming that 2018 usage indicates typical GBT usage, we get about 123 days of observations at the maximum sensitivity. Even if half of these data are corrupted by Radio-frequency Interference (RFI), a non-detection would exclude all values of $\alpha>1$. A limited number of detections in the first year would also help putting useful constraints on the values of $R_{0}$, which is the instrument-independent all-sky FRB rate. Thus, GREENBURST is a promising FRB instrument regardless of the outcome.

Most current FRB back-ends search multiple beams (e.g. ASKAP, CHIME, Parkes, ALFABURST etc.), while GREENBURST is a single-beam survey. Although this reduces sky coverage, the high sensitivity of the GBT still means it has the potential to detect FRBs. This increases the search volume in redshift and thus compensates for the lack of sky coverage. If a repeating FRB were detected with GREENBURST, the narrow telescope beam would reduce the prospective sky area for followup observations. This would speed-up the identification of the host galaxy associated with the FRB.

The GREENBURST pipeline will carry out a blind search for single-pulses over a large DM range. This range includes Galactic DMs as well. Thus, in addition to FRBs, our pipeline may also discover radio transients in our Galaxy. The distinct advantage provided by the commensal mode observing is that GREENBURST would be recording data all the time, enabling it to make serendipitous discovery of a Galactic radio transient. We have already made such a discovery with the ALFABURST while the telescope was slewing between targets (Foster et al., 2018). In addition, GREENBURST may detect more radio pulses from known rotating radio transients (RRATs) ${ }^{6}$.

We have a working pipeline, and at the time of writing, have acquired data for a week. We are currently carrying out data quality checks and tuning our search pipeline to the observed RFI in the data. We are also ascertaining whether all the metadata are properly logged by crossreferencing them with the GBT logs. GREENBURST is exiting its commissioning phase and would soon start searching for FRBs in real-time.

\section{ACKNOWLEDGEMENTS}

We thank West Virginia University for its financial support of GBT operations, which enabled some of the observations for this project. M.P.S., M.A.M. and D.R.L. acknowledge support from NSF RII Track I award number OIA-1458952. M.P.S., M.A.M., G.G. and D.R.L. are members of the NANOGrav Physics Frontiers Center which is supported by NSF award 1430284. Berkeley efforts were supported by NSF grants 1407804 and 1711254, as well as the Marilyn and Watson Alberts SETI Chair funds. The Green Bank Observatory is a facility of the National Science Foundation operated under cooperative agreement by Associated Universities, Inc.

\section{REFERENCES}

Bhandari S., et al., 2018, MNRAS, 475, 1427

CHIME/FRB Collaboration et al., 2019a, Nature, 566, 230

CHIME/FRB Collaboration et al., 2019b, Nature, 566, 235

Chawla P., et al., 2017, ApJ, 844, 140

Chennamangalam J., et al., 2017, ApJS, 228, 21

Foster G., et al., 2018, MNRAS, 474, 3847

Gajjar V., et al., 2018, ApJ, 863, 2

Golpayegani G., Lorimer D., 2019, in American Astronomical Society Meeting Abstracts \#233. p. 110.01

Lawrence E., Vander Wiel S., Law C., Burke Spolaor S., Bower G. C., 2017, AJ, 154, 117

Lorimer D. R., Bailes M., McLaughlin M. A., Narkevic D. J., Crawford F., 2007, Science, 318, 777

Maddalena R. J., 2013, Technical Report 285, Properties of the GBT at L-band for Commensal Observing, https://library.nrao.edu/public/ memos/gbt/GBT_285.pdf. NRAO, Green Bank, WV, https://library.nrao.edu/public/memos/gbt/ GBT_285.pdf

Marcote B., et al., 2017, ApJ, 834, L8

Masui K., et al., 2015, Nature, 528, 523

Michilli D., et al., 2018, Nature, 553, 182

Petroff E., et al., 2016, PASA, 33, e045

Shannon R. M., et al., 2018, Nature, 562, 386

Spitler L. G., et al., 2016, Nature, 531, 202

\footnotetext{
${ }^{6}$ http://astro.phys.wvu.edu/rratalog
} 
Thornton D., et al., 2013, Science, 341, 53

Zhang Y. G., Gajjar V., Foster G., Siemion A., Cordes J., Law C., Wang Y., 2018, ApJ, 866, 149 